This article was downloaded by: [Inra]

On: 05 J une 2014, At: 07:56

Publisher: Taylor \& Francis

Informa Ltd Registered in England and Wales Registered Number: 1072954 Registered office: Mortimer House, 37-41 Mortimer Street, London W1T 3J H, UK

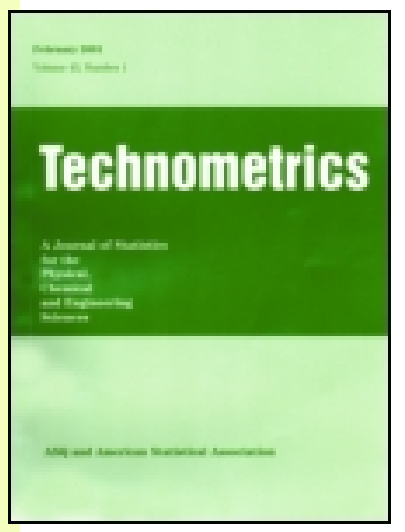

\title{
Technometrics
}

Publication details, including instructions for authors and subscription information: http:// amstat. tandfonline.com/loi/ utch20

\section{Quantile-Based Optimization of Noisy Computer Experiments With Tunable Precision}

\author{
Victor Picheny ${ }^{a}$, David Ginsbourger ${ }^{b}$, Yann Richet ${ }^{c} \&$ Gregory Caplin ${ }^{c}$ \\ ${ }^{a}$ Centre Européen de Recherche et de Formation Avance en Calcul Scientifique , 31057 , \\ Toulouse, France \\ ${ }^{\mathrm{b}}$ Department of Mathematics and Statistics, University of Bern, 3012, Bern, \\ Switzerland \\ c Institut de Radioprotection et de Sûreté Nucléaire , 92260, Fontenay-aux-Roses, \\ France \\ Accepted author version posted online: 11 J ul 2012. Published online: 22 Feb 2013.
}

To cite this article: Victor Picheny, David Ginsbourger , Yann Richet \& Gregory Caplin (2013) Quantile-

Based Optimization of Noisy Computer Experiments With Tunable Precision, Technometrics, 55:1, 2-13, DOI:

10.1080/00401706.2012.707580

To link to this article: http:// dx.doi.org/ 10.1080/00401706.2012.707580

\section{PLEASE SCROLL DOWN FOR ARTICLE}

Taylor \& Francis makes every effort to ensure the accuracy of all the information (the "Content") contained in the publications on our platform. However, Taylor \& Francis, our agents, and our licensors make no representations or warranties whatsoever as to the accuracy, completeness, or suitability for any purpose of the Content. Any opinions and views expressed in this publication are the opinions and views of the authors, and are not the views of or endorsed by Taylor \& Francis. The accuracy of the Content should not be relied upon and should be independently verified with primary sources of information. Taylor and Francis shall not be liable for any losses, actions, claims, proceedings, demands, costs, expenses, damages, and other liabilities whatsoever or howsoever caused arising directly or indirectly in connection with, in relation to or arising out of the use of the Content.

This article may be used for research, teaching, and private study purposes. Any substantial or systematic reproduction, redistribution, reselling, loan, sub-licensing, systematic supply, or distribution in any form to anyone is expressly forbidden. Terms \& Conditions of access and use can be found at http:// amstat.tandfonline.com/page/terms-and-conditions 


\title{
Quantile-Based Optimization of Noisy Computer Experiments With Tunable Precision
}

\author{
Victor PICHENY \\ Centre Européen de Recherche et de Formation \\ Avance en Calcul Scientifique \\ 31057 Toulouse, France \\ (picheny@cerfacs.fr)
}

\author{
David GinsBOURGER \\ Department of Mathematics and Statistics \\ University of Bern, 3012 Bern \\ Switzerland \\ (david.ginbourger@stat.unibe.ch)
}

\author{
Yann RICHET and Gregory CAPLIN \\ Institut de Radioprotection et de Sûreté Nucléaire \\ 92260 Fontenay-aux-Roses \\ France \\ (yann.richet@irsn.fr, gregory.caplin@irsn.fr)
}

\begin{abstract}
This article addresses the issue of kriging-based optimization of stochastic simulators. Many of these simulators depend on factors that tune the level of precision of the response, the gain in accuracy being at a price of computational time. The contribution of this work is two-fold: first, we propose a quantile-based criterion for the sequential design of experiments, in the fashion of the classical expected improvement criterion, which allows an elegant treatment of heterogeneous response precisions. Second, we present a procedure for the allocation of the computational time given to each measurement, allowing a better distribution of the computational effort and increased efficiency. Finally, the optimization method is applied to an original application in nuclear criticality safety. This article has supplementary material available online. The proposed criterion is available in the R package DiceOptim.
\end{abstract}

KEY WORDS: Expected improvement; Kriging; Stochastic simulators.

\section{INTRODUCTION}

Using metamodels for facilitating optimization and statistical analysis of computationally expensive simulators has become commonplace. In particular, the kriging-based efficient global optimization (EGO) algorithm (Jones, Schonlau, and Welch 1998) has been recognized as an efficient tool for deterministic black-box optimization.

The way a simulator response follows the function of interest is called fidelity. Oftentimes, a large range of response fidelities is available by tuning factors that control the complexity of numerical methods. For instance, the precision of a finite element analysis can be controlled by the discretization technique or the solver convergence. When the response stems from Monte Carlo methods (which are often referred to as stochastic simulators), accuracy (measured by the inverse of the response variance) is proportional to sample size. Such simulators are often called noisy simulators, since they return approximate solutions that depart from the exact value by an error term that can be considered as a random quantity.

In an optimization context, having noise in the responses requires a proper adaptation of criteria and algorithms. Furthermore, for each simulation run, the user has to set a trade-off between computational cost and response precision. This additional degree of freedom may greatly improve the efficiency of the optimization but requires appropriate tools to choose this trade-off and the ability to work with heterogeneous precisions.

Using metamodels for noisy optimization has been addressed by several authors. Huang et al. (2006) and Forrester, Keane, and Bressloff (2006) proposed kriging-based strategies for opti- mization of uniformly noisy functions. However, little work can be found in the case of heterogeneous noise. Most approaches combining optimization and variable precision are found in the multifidelity framework (Gano et al. 2006; Forrester, Sóbester, and Keane 2007) but consider only two fidelity levels, the lowfidelity model being used as a helping tool to choose the highfidelity evaluations.

This article proposes two contributions to this framework. First, we define an extension of expected improvement (EI) based on quantiles that enables an elegant treatment of both continuous or discrete fidelities. The proposed criterion depends not only on the noise variances from the past, but also on the fidelity of the new candidate measurement. Second, we study a procedure taking advantage of the possibility to choose the fidelity level at each iteration.

In the next section, we define the "noisy" framework we are considering and present briefly the kriging model. Section 3 describes the classical kriging-based optimization procedure and its limitation with noisy functions. In Section 4, we propose a new infill criterion, called Expected Quantile Improvement (EQI), well-suited for the noisy framework, and in Section 5, we propose a numerical trick for tuning one of the EQI parameters to account for finite computational budgets. Section 6 describes a procedure for online decision on precision level. Finally, this

C 2013 American Statistical Association and the American Society for Quality TECHNOMETRICS, FEBRUARY 2013, VOL. 55, NO. 1 DOI: 10.1080/00401706.2012.707580 
procedure is compared with existing kriging-based methods and applied to an original application in nuclear criticality safety.

\section{NOTATIONS AND CONCEPTS}

\subsection{The Noisy Optimization Problem}

We consider a single objective, unconstrained minimization problem over a compact set $D$. The deterministic objective function $y: \mathbf{x} \in D \subset \mathbb{R}^{d} \longrightarrow y(\mathbf{x}) \in \mathbb{R}$ is here observed with noise, that is, the user only has access to measurements of the form $\widetilde{y_{i}}=y\left(\mathbf{x}^{i}\right)+\epsilon_{i}$, where $\epsilon_{i}$ is assumed to be one realization of a "noise" random variable $\varepsilon$. In the rest of this article, we make the assumption that the observation noises are normally distributed, centered, and independent from one run to each other: $\varepsilon_{i} \sim \mathcal{N}\left(0, \tau_{i}^{2}\right)$ independently.

\subsection{Noise in Computer Experiments}

In classical experiments, noise usually accounts for a large number of uncontrolled variables (variations of the experimental setup, measurement precision, etc.). In computer experiments, noise can have many sources, including modeling and discretization error, incomplete convergence, and finite sample size for Monte Carlo methods, see for instance Forrester, Keane, and Bressloff (2006) or Gramacy and Lee (2010a) for a detailed discussion.

The nature of the noise depends on the associated simulator. When classical Monte Carlo simulations are involved in the output evaluation, error is independent from one run to each other, even for measurements with the same input variables. Such simulators are often referred to as stochastic, and are the main target for the method presented here. The industrial application described in Section 7.2 belongs to this category.

Errors due to a simplification of the physics, geometry, or meshing tend to show strong correlations, especially for simulations with similar fidelities, and repeated experiments provide the same observations. This situation has been addressed in the multifidelity literature [see Kennedy and O'Hagan (2000) and Qian and Wu (2008) for modeling; Forrester, Sóbester, and Keane (2007) and Huang et al. (2006) for optimization] and is not considered here, although many of the concepts presented here may apply with a proper adaptation of the kriging model.

Error due to incomplete convergence can be either treated as correlated noise or not. In Forrester, Bressloff, and Keane (2006), it is observed that simulations tend to converge in unison, which makes the partial convergence equivalent to a multifidelity problem. However, when the output convergence behavior varies substantially across the design space, the hypothesis of independence of the error between runs may become reasonable, especially if experiments are well spread in the design space and different convergence levels are used.

\subsection{Experiments With Tunable Precision}

As mentioned in the introduction, the precision of many simulators can be tuned by the user, for instance by changing the number of solver steps for incomplete convergence or the sample size for Monte Carlo methods. Here, we consider that, for every measurement, the noise variance $\tau_{i}^{2}=\tau^{2}\left(t_{i}\right)$ is a monotonically decreasing function of computation time $t_{i}$.

A perhaps "canonical" example of tunable precision is when the response considered is obtained by averaging an arbitrary number $b_{i}$ of independent drawings (which is a typical situation in the framework of robust optimization for instance):

$$
\widetilde{Y}_{i}=\frac{1}{b_{i}} \sum_{j=1}^{b_{i}} y\left(\mathbf{x}_{i}\right)+\varepsilon_{i, j},
$$

when $\varepsilon_{i, j} \sim \mathcal{N}\left(0, v^{2}\right)$. We have then $\tilde{Y}_{i} \sim \mathcal{N}\left(y\left(\mathbf{x}_{i}\right), \frac{v^{2}}{b_{i}}\right)$, so $\tau^{2}(t)=\rho v^{2} / t, \rho$ being the time needed for a single drawing. The value of $b_{i}$ chosen by the user tunes the precision of $\widetilde{Y}_{i}$.

In this work, we make two strong assumptions: (a) the computation time, and hence the error variance, is controllable and (b) the function $\tau(t)$ is accurately known. Although some stochastic simulators, such as the one described in Section 7.2, directly provide an accurate estimate of the output uncertainty, in most real applications, a learning study is necessary, typically assuming a (simple) parametric form for the variance. In the case of Monte Carlo simulators and assuming small variations of the output across the design space, we have $\tau^{2}(t)=C / t$, where $C$ is an unknown constant that can be estimated when building the kriging model, as described in Section 2.4.

Finally, for simulators relying on Monte Carlo or on iterative solvers, the response corresponding to a given precision is not obtained directly but more as a limit of intermediate responses of lower precisions. For each measurement, the noisy response $\tilde{y}_{i}$ is thus obtained as the last term of a sequence of measurements $\tilde{y}_{i}[1], \ldots, \tilde{y}_{i}\left[b_{i}\right]$, where $b_{i} \in \mathbb{N}$ is the number of calculation steps at the $i$ th measurement.

Figure 1 represents two examples of response convergence. First, the convergence of the output of the stochastic simulator of Section 7.2 is drawn for its nominal design values. Here, the variance is known accurately, and depicted by the $90 \%$ confidence interval. The curve $\tilde{y}$ represents the sequence $\tilde{y}[j], j=1, \ldots, 100$. The second figure is taken from Forrester, Bressloff, and Keane (2006) and represents the convergence of an objective function (namely the L/D ratio) calculated using an Euler simulation of an aerofoil, as a function of the number of solver steps. The response oscillates around its final value with decreasing amplitude. Here, error variance is not available directly and requires the specification of and inferences for a parametric model for $\tau$ based on a couple of trial responses such as this one.

\subsection{The Kriging Metamodel}

In this work, we use a (generalized) Gaussian process (GP) regression model [as in Rasmussen and Williams (2006), chap. 2], where $y$ is assumed to be one realization of a random process $Y$ with an unknown constant trend $\mu \in \mathbb{R}$, and a stationary covariance kernel $k$, that is, of the form $k:\left(\mathbf{x}, \mathbf{x}^{\prime}\right) \in D^{2} \longrightarrow k\left(\mathbf{x}, \mathbf{x}^{\prime}\right)=$ $\sigma^{2} r\left(\mathbf{x}-\mathbf{x}^{\prime} ; \psi\right)$ for some admissible correlation function $r$ with parameters $\psi$. Provided that the process $Y$ and the Gaussian measurement errors $\varepsilon_{i}$ are stochastically independent and that the error variances are given, the distribution of $Y(\mathbf{x})$ conditional 

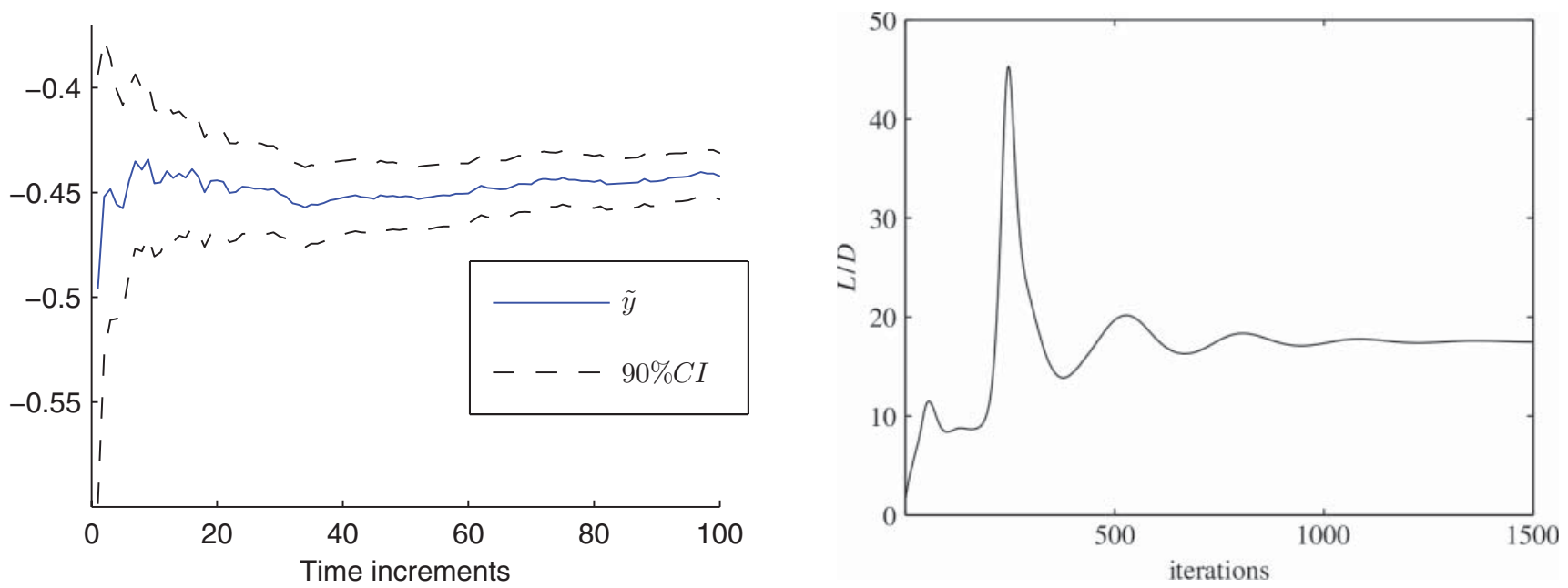

Figure 1. Examples of tunable precision responses. Left: convergence of the output of MORET for its nominal design values; right: partially converged response of a computational fluid dynamics (CFD) code. The online version of this figure is in color.

on the event $\widetilde{A_{n}}=\left\{Y\left(\mathbf{x}^{i}\right)+\varepsilon_{i}=\widetilde{y_{i}}, 1 \leq i \leq n\right\}$ is

$$
Y(\mathbf{x}) \mid \widetilde{A_{n}} \sim \mathcal{N}\left(m_{n}(\mathbf{x}), s_{n}^{2}(\mathbf{x})\right),
$$

where $m_{n}($.$) and s_{n}^{2}($.$) are, respectively, the kriging mean and$ variance, given by

$$
\begin{aligned}
m_{n}(\mathbf{x})= & \widehat{\mu}_{n}+\mathbf{k}_{n}(\mathbf{x})^{T}\left(K_{n}+\Delta_{n}\right)^{-1}\left(\widetilde{\mathbf{y}}^{n}-\widehat{\mu}_{n} \mathbf{1}_{n}\right), \\
s_{n}^{2}(\mathbf{x})= & \sigma^{2}-\mathbf{k}_{n}(\mathbf{x})^{T}\left(K_{n}+\Delta_{n}\right)^{-1} \mathbf{k}_{n}(\mathbf{x}) \\
& +\frac{\left(1-\mathbf{1}_{n}^{T}\left(K_{n}+\Delta_{n}\right)^{-1} \mathbf{k}_{n}(\mathbf{x})\right)^{2}}{\mathbf{1}_{n}^{T}\left(K_{n}+\Delta_{n}\right)^{-1} \mathbf{1}_{n}}
\end{aligned}
$$

with $\sigma^{2}=k(\mathbf{x}, \mathbf{x}), \widetilde{\mathbf{y}}^{n}=\left(\widetilde{y}_{1}, \ldots, \widetilde{y}_{n}\right)^{T}, K_{n}=\left(k\left(\mathbf{x}^{i}, \mathbf{x}^{j}\right)\right)_{1 \leq i, j \leq n}$, $\mathbf{k}_{n}(\mathbf{x})=\left(k\left(\mathbf{x}, \mathbf{x}^{1}\right), \ldots, k\left(\mathbf{x}, \mathbf{x}^{n}\right)\right)^{T}, \Delta_{n}$ is a diagonal matrix of diagonal terms $\tau_{1}^{2}, \ldots, \tau_{n}^{2}, \mathbf{1}_{n}$ is an $n \times 1$ vector of ones, and $\widehat{\mu}_{n}=\mathbf{1}_{n}^{T}\left(K_{n}+\Delta_{n}\right)^{-1} \widetilde{\mathbf{y}}^{n} / \mathbf{1}_{n}^{T}\left(K_{n}+\Delta_{n}\right)^{-1} \mathbf{1}_{n}$ is the best linear unbiased estimate of $\mu$.

As for a classical kriging model, the covariance parameters $\sigma^{2}$ and $\psi$ usually need to be estimated, using maximum likelihood (ML) for instance and considering the noise variances as known. If the noise variances are not known but a simple parametric functional relashionship is assumed between the $\tau_{i}^{2}$ 's and the $t_{i}$ 's, the corresponding parameters may be embedded within the ML procedure. For instance, assuming a Monte Carlo type behavior of the form $\tau_{i}^{2}=C / t_{i}$, the likelihood would depend on $C$ through $\Delta_{n}$ and $\widehat{\mu}_{n}$.

\section{KRIGING-BASED OPTIMIZATION; LIMITATIONS WITH NOISY FUNCTIONS}

The EGO algorithm (Jones, Schonlau, and Welch 1998) builds a sequential design with the goal of finding a global minimum of a black-box function. It consists of sequentially evaluating $y$ at a point maximizing a figure of merit relying on kriging, the EI criterion, and updating the metamodel after each new observation.

In the noiseless case, with $y_{i}=y\left(\mathbf{x}^{i}\right)(1 \leq i \leq n), \mathbf{y}^{n}=$ $\left(y_{1}, \ldots, y_{n}\right)^{T}, \mathbf{X}^{n}=\left\{\mathbf{x}^{1}, \ldots, \mathbf{x}^{n}\right\}$, and $A_{n}$ denoting the event $Y\left(\mathbf{X}^{n}\right)=\mathbf{y}^{n}$, the improvement provided by sampling at $\mathbf{x}$ is defined by $I=\max \left(0, \min \left(Y\left(\mathbf{X}^{n}\right)\right)-Y(\mathbf{x})\right)$, and the EI is its Comment citer ce documer expectation given by the GP model:

$$
\begin{aligned}
\mathrm{EI}_{n}(\mathbf{x}) & :=\mathbb{E}\left[\left(\min \left(Y\left(\mathbf{X}^{n}\right)\right)-Y(\mathbf{x})\right)^{+} \mid A_{n}\right] \\
& =\mathbb{E}\left[\left(\min \left(\mathbf{y}^{n}\right)-Y(\mathbf{x})\right)^{+} \mid A_{n}\right],
\end{aligned}
$$

An integration by parts yields the well-known analytical expression:

$$
\begin{aligned}
\mathrm{EI}_{n}(\mathbf{x}):= & \left(\min \left(\mathbf{y}^{n}\right)-m_{n}(\mathbf{x})\right) \Phi\left(\frac{\min \left(\mathbf{y}^{n}\right)-m_{n}(\mathbf{x})}{s_{n}(\mathbf{x})}\right) \\
& +s_{n}(\mathbf{x}) \phi\left(\frac{\min \left(\mathbf{y}^{n}\right)-m_{n}(\mathbf{x})}{s_{n}(\mathbf{x})}\right),
\end{aligned}
$$

where $\Phi$ and $\phi$ are, respectively, the cumulative distribution function and the probability density function of the standard Gaussian law. The latter analytical expression is very convenient since it allows fast evaluations of EI, and even analytical calculation of its gradient. Now, in the context of noisy evaluations, (5) is not very satisfactory for at least two reasons. First, the current minimum $\min \left(Y\left(\mathbf{X}^{n}\right)\right)$ is not deterministically known conditionally on the noisy observations, unlike the noiseless case. Second, the EI is based on the improvement produced by a deterministic evaluation of $y$ at the candidate point $\mathbf{x}$. Now, if the next evaluation is noisy, $Y(\mathbf{x})$ will remain inexactly known. It would hence benefit from a new criterion taking the precision of the next measurement into account.

In Huang et al. (2006), a heuristic modification of the EI called Augmented Expected Improvement (AEI) is proposed for uniformly noisy observations. The mean predictor at the training point with smallest kriging quantile is used as a surrogate value for $\min \left(Y\left(\mathbf{X}^{n}\right)\right)$, and the EI is multiplied by a penalization function $1-\tau / \sqrt{s_{n}^{2}(\mathbf{x})+\tau^{2}}$ to limit replications.

A more rigorous alternative, as noted by Gramacy and Lee (2010b) and Gramacy and Polson (2011), consists of computing the EI based on the joint distribution of $\left(\min \left(Y\left(\mathbf{X}^{n}\right)\right), Y(\mathbf{x})\right)$ conditional on $\widetilde{A_{n}}$; however, in this form, the EI must be estimated by expensive Monte Carlo simulations, which makes the EI maximization challenging.

Finally, the integrated expected conditional improvement (IECI), proposed by Gramacy and Lee (2010b), evaluates by how much a candidate measurement at a given point would 
affect the EI over the design space, thereby naturally taking past and future noises into account. However, this criterion requires a numerical integration over the design space, which can be time consuming, especially in high dimensions.

The next section presents a class of criteria (indexed by a parameter $\beta$ tuning a quantile level) that takes into account past and future noises with transparent probabilistic foundations and that can be derived analytically as a function of the future point and its associated noise level.

\section{EXPECTED QUANTILE IMPROVEMENT}

\subsection{Definition}

Our aim is to get a kriging-based optimization criterion measuring which level of improvement can be statistically expected from evaluating $y$ at a new $\mathbf{x}$ with a noise of given variance $\tau^{2}$. A first question to be addressed is of decision-theoretical nature: what does the term "improvement" mean when comparing two sets of noisy observations? What criterion should be used to judge that a set of noisy observations, or the associated metamodel, is better (in terms of minimization) after the $(n+1)$ th measurement than before it?

Using only the noisy observations $\widetilde{\mathbf{y}}^{n}$ and $\widetilde{y}^{n+1}$ is a highly risky strategy, since the noise may introduce errors in the ranking of the observations. Here we propose to use the $\beta$-quantiles given by the kriging conditional distribution, for a given level $\beta \in[0.5,1)$ : a point is declared "best" over a set of candidates $\mathbf{X}^{n}$ whenever it has the lowest $\beta$-quantile:

$$
\begin{aligned}
\mathbf{x}^{*} & =\arg \min _{\mathbf{x} \in \mathbf{X}_{n}}\left[q_{n}(\mathbf{x})\right] \\
& =\arg \min _{\mathbf{x} \in \mathbf{X}_{n}}\left[m_{n}(\mathbf{x})+\Phi^{-1}(\beta) s_{n}(\mathbf{x})\right] .
\end{aligned}
$$

This is the criterion also considered by Huang et al. (2006).

Now, we propose to define an improvement that is consistent with our decision criterion: we define improvement $I$ to be the decrease of the lowest $\beta$-quantile, between the present step $n$ and the forthcoming step $n+1$ :

$$
I=\left(\min \left(q_{n}\left(\mathbf{X}^{n}\right)\right)-q_{n+1}\left(\mathbf{x}^{n+1}\right)\right)^{+} .
$$

Of course, like in the noiseless case, this improvement cannot be known in advance, because $q_{n+1}\left(\mathbf{x}_{n+1}\right)$ depends on the future observation $\tilde{y}_{n+1}$. However, thanks to the particular form of the kriging equations, the future quantile $q_{n+1}$ can be predicted, and consequently the EI can be calculated, based on the GP model at step $n$, as we show below.

In our improvement (8), we restrict attention to the observed points $\left(\mathbf{X}^{n}\right.$ and $\left.\mathbf{x}^{n+1}\right)$, even though a similar criterion could be defined over the entire design space:

$$
I=\left(\min _{D}\left(q_{n}(\mathbf{x})\right)-\min _{D}\left(q_{n+1}(\mathbf{x})\right)\right)^{+}
$$

However, such a restriction allows simplification, yielding a criterion in closed form.

Let us denote by $Q_{i}(\mathbf{x})$ the kriging quantile $q_{i}(\mathbf{x})(i \leq n+1)$ where the measurements are still in their random form, and define the EQI as

$\mathrm{EQI}_{n}\left(\mathbf{x}^{n+1}, \tau_{n+1}^{2}\right):=\mathbb{E}\left[\left(\min _{i \leq n}\left(Q_{n}\left(\mathbf{x}^{i}\right)\right)-Q_{n+1}\left(\mathbf{x}^{n+1}\right)\right)^{+} \mid \widetilde{A_{n}}\right]$,

where the dependence on the future noise $\tau_{n+1}^{2}$ appears through $Q_{n+1}(\mathbf{x})$ 's distribution.

The randomness of $Q_{n+1}(\mathbf{x})$ conditional on $\widetilde{A_{n}}$ is indeed a consequence from $\widetilde{Y_{n+1}}:=Y\left(\mathbf{x}^{n+1}\right)+\varepsilon_{n+1}$ having not been observed yet at step $n$. However, following the fact that $\widetilde{Y_{n+1}} \mid \widetilde{A_{n}}$ is Gaussian with known mean and variance, one can show that $Q_{n+1}($.$) is a GP conditional on \widetilde{A_{n}}$ (see proof and details in the Appendix). Furthermore, $\min _{i \leq n}\left(Q_{n}\left(\mathbf{x}^{i}\right)\right)$ is known conditional on $\widetilde{A_{n}}$. As a result, the proposed EQI is analytically tractable, and we get by a similar calculation as in (6):

$$
\begin{aligned}
\operatorname{EQI}_{n}\left(\mathbf{x}^{n+1}, \tau_{n+1}^{2}\right)= & \left(\min \left(\mathbf{q}^{n}\right)-m_{Q_{n+1}}\right) \Phi\left(\frac{\min \left(\mathbf{q}^{n}\right)-m_{Q_{n+1}}}{s_{Q_{n+1}}}\right) \\
& +s_{Q_{n+1}} \phi\left(\frac{\min \left(\mathbf{q}^{n}\right)-m_{Q_{n+1}}}{s_{Q_{n+1}}}\right),
\end{aligned}
$$

where $\mathbf{q}^{n}:=\left\{q_{n}\left(\mathbf{x}^{i}\right), i \leq n\right\}$ is the set of current quantile values at the already visited points, $m_{Q_{n+1}}:=\mathbb{E}\left[Q_{n+1}\left(\mathbf{x}^{n+1}\right) \mid \widetilde{A_{n}}\right]$ is $Q_{n+1}\left(\mathbf{x}^{n+1}\right)$ 's conditional expectation-seen from step $n$, and $s_{Q_{n+1}}^{2}:=\operatorname{var}\left[Q_{n+1}\left(\mathbf{x}^{n+1}\right) \mid \widetilde{A_{n}}\right]$ is its conditional variance, both derived in the Appendix.

\subsection{Properties}

The EQI criterion has the following important properties:

(a) In absence of future noise $\left(\tau_{n+1}^{2}=0\right)$, the future quantile at $\mathbf{x}^{n+1}$ coincides with the observation $\tilde{y}^{n+1}=y\left(\mathbf{x}^{n+1}\right)$; it follows directly that $Q_{n+1}\left(\mathbf{x}^{n+1}\right)\left|\widetilde{A_{n}}=Y_{n+1}\right| \widetilde{A_{n}}$, so the $\mathrm{EQI}$ is equal to the classical EI with a plugin of the kriging quantiles for $\min \left(\mathbf{y}^{n}\right)$.

(b) In absence of past noise (for the $n$ first observations), $\min \left(\mathbf{q}^{n}\right)$ is equal to the minimum of the observations, $\min \left(\mathbf{y}^{n}\right)$.

(c) In absence of both past and future noises, the EQI is then equal to the classical EI.

The parameter $\beta$ tunes the level of reliability wanted on the final result [which plays a similar role as the power parameter of the generalized improvement of Schonlau, Welch, and Jones (1998)]. With $\beta=0.5$, the design points are compared based on the kriging mean predictor only, without taking into account the prediction variance at those points, while high values of $\beta$ (i.e., near to 1) penalize designs with high uncertainty, which is a more conservative approach. Hence, with a high $\beta$, the criterion is more likely to favor observation repetitions or clustering, to locally decrease the prediction variance, while with $\beta=0.5$, the criterion can be expected to be more exploratory.

The future noise $\tau_{n+1}^{2}$ also strongly affects the shape of the EQI. Indeed, a very noisy future observation can only have a very limited influence on the kriging model. Then, the only way to get a nonzero improvement is either to sample where $q_{n}(\mathbf{x})$ is minimum if this point is not in $\mathbf{X}^{n}$ (the lowest quantile will then be chosen on the set $\mathbf{X}^{n+1}$ instead of $\mathbf{X}^{n}$, which will bring an 

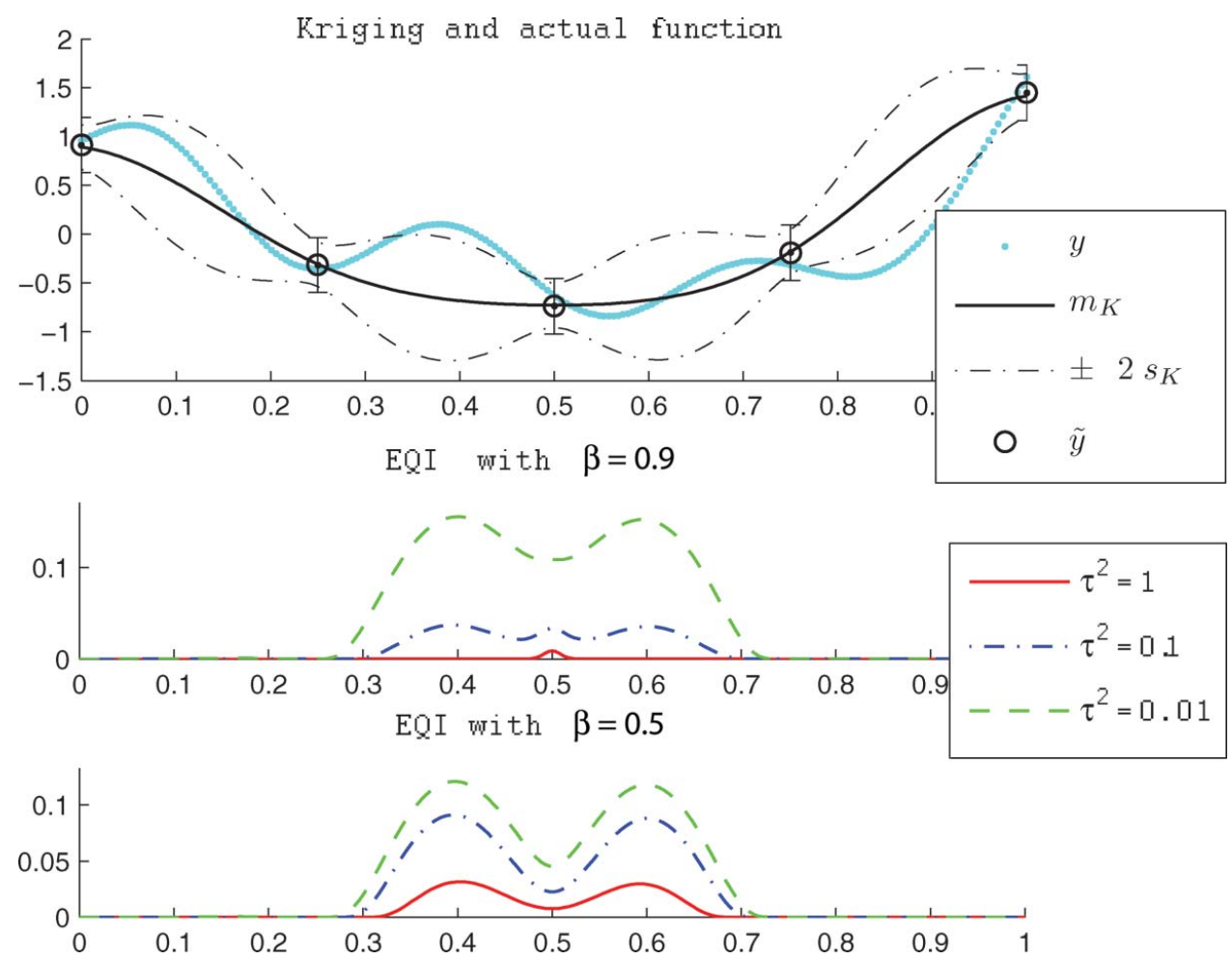

Figure 2. Actual function, kriging, and corresponding EQI for three future noise levels and two quantile levels. The bars of the upper graph show the noise amplitude $\left( \pm 2 \times \tau_{i}\right)$. The online version of this figure is in color.

improvement even if $q_{n+1}=q_{n}$ ), or to sample at the current best point, which may decrease its uncertainty and brings a small but measurable improvement. On the contrary, if $\tau_{n+1}^{2}$ is very small, the EQI behaves like the classical EI, making the well-known trade-off between exploration and exploitation.

Figure 2 illustrates the dependence of the EQI on both $\tau_{n+1}^{2}$ and $\beta$. The actual function is $y(x)=\frac{1}{2}(\sin (20 x) /(1+x)+$ $\left.3 x^{3} \cos (5 x)+10(x-0.5)^{2}-0.6\right)$, the initial design consists of five equally spaced measurements, with noise variances equal to 0.02 .

We can see that the choice of the future noise level has a substantial influence on the criterion. With small noise variance, the EQI behaves like the classical EI, with highest values in regions with high uncertainty and low mean predictions. With higher noise variances and high quantiles, the criterion becomes very conservative since it is high only in the vicinity of existing measurements. With $\beta=0.5$, the $\mathrm{EQI}$ is high even for the largest variance. However, here five curves out of six return similar optimal locations (i.e., $\mathbf{x}$ near 0.4 or 0.6 ), which indicates that the influence of $\tau$ and $\beta$ may not be critical during the first optimization steps.

\subsection{EQI as a Function of Computational Time}

EQI measures the effect of a new measurement with variance $\tau_{n+1}^{2}$, while we defined in Section 2 a measurement at location $\mathbf{x}_{i}$ as depending on a computational time $t_{i}$. In our framework of tunable precision, two cases have to be distinguished. Let $t_{n+1}$ be the computational time used at iteration $n+1$. At unsampled locations, the EQI criterion is simply evaluated with $\tau_{n+1}^{2}=\tau^{2}\left(t_{n+1}\right)$. At existing observations, a different value has to be used, if not, the EQI would estimate the value of a new measurement with variance $\tau^{2}\left(t_{n+1}\right)$, instead of estimating the value of improving the existing measurement. To compute this value, we use the fact that it is equivalent for the kriging model to have several measurements at the same point with independent noises or a single equivalent measurement which is the weighted average of the observations (see the online supplementary material for proof). For instance, let $\tilde{y}_{i, 1}$ and $\tilde{y}_{i, 2}$ be two measurements with noise levels $\tau_{i, 1}^{2}$ and $\tau_{i, 2}^{2}$, respectively. They are equivalent to

$$
\tilde{y}_{i, e q}=\frac{\tau_{i, 1}^{-2} \tilde{y}_{i, 1}+\tau_{i, 2}^{-2} \tilde{y}_{i, 2}}{\tau_{i, 1}^{-2}+\tau_{i, 2}^{-2}},
$$

with variance $\tau_{i, e q}^{2}$ the harmonic mean of $\tau_{i, 1}^{2}$ and $\tau_{i, 2}^{2}$, namely,

$$
\frac{1}{\tau_{i, e q}^{2}}:=\frac{1}{\tau_{i, 1}^{2}}+\frac{1}{\tau_{i, 2}^{2}} \Longrightarrow \tau_{i, e q}^{2}=\frac{\tau_{i, 1}^{2} \tau_{i, 2}^{2}}{\tau_{i, 1}^{2}+\tau_{i, 2}^{2}} .
$$

Now, we want to measure the effect of improving a measurement with initial error variance $\tau^{2}\left(t_{i}\right)$ until the variance $\tau^{2}\left(t_{i}+t_{n+1}\right)$ is reached. This is equivalent, in terms of the kriging model, to taking a new measurement with noise variance:

$$
\tau^{2}\left(t_{i} \rightarrow t_{i}+t_{n+1}\right):=\frac{\tau^{2}\left(t_{i}\right) \tau^{2}\left(t_{i}+t_{n+1}\right)}{\tau^{2}\left(t_{i}\right)-\tau^{2}\left(t_{i}+t_{n+1}\right)} .
$$

This formula is obtained from (12), with $\tau_{i, e q}^{2}=\tau^{2}\left(t_{i}+t_{n+1}\right)$, $\tau_{i, 1}^{2}=\tau^{2}\left(t_{i}\right)$ and $\tau_{i, 2}^{2}=\tau^{2}\left(t_{i} \rightarrow t_{i}+t_{n+1}\right)$. Hence, at $\mathbf{x}^{i}$, EQI may be evaluated with $\tau_{n+1}^{2}=\tau^{2}\left(t_{i} \rightarrow t_{i}+t_{n+1}\right)$.

The next section proposes a numerical trick for tuning $t_{n+1}$ to account for finite computational budgets. 


\section{OPTIMIZATION WITH FINITE COMPUTATIONAL BUDGET}

It is well known that the EGO algorithm is a so-called myopic strategy, since its criterion EI always considers the next step as if it were the last one. However, for most computer experiments, the total computational budget is bounded and prescribed by industrial constraints such as time and power limitations. In the deterministic framework, this results in a limited (given) number of observations for optimization. It has been shown [Mockus (1988) followed by Ginsbourger and Le Riche (2010)] that taking into account the finite budget may modify the optimization strategy and improve significantly its efficiency.

Here, the concept of finite budget is particularly critical, since each observation requires a trade-off between accuracy and rapidity, and in general, the user has to trade-off between the total number of observations and their precision. In linear modeling, this problem is typical of the theory of optimal designs (Fedorov and Hackl 1997), with the notable difference that we face it here within a sequential strategy, and a nonlinear (kriging) model.

The computational constraint implies that the sum of all computational times is fixed to a given budget, say $T_{0}$. At step $n$, the remaining budget for optimization is $T_{n+1}=T_{0}-\sum_{i=1}^{n} t_{i}$.

The EQI criterion allows taking into account such computational budget in the choice of the new candidate observations. Indeed, the future noise level $\tau_{n+1}^{2}$, which is a parameter of EQI, will stand here for the finite budget. Given a computational budget $T_{n+1}$, the smallest noise variance achievable (i.e., the largest precision) for a new measurement is $\tau^{2}\left(T_{n+1}\right)$, and $\tau^{2}\left(t_{i} \rightarrow t_{i}+T_{n+1}\right)$ at $\mathbf{x}^{i}(1 \leq i \leq n)$, assuming that all the remaining budget will be attributed to this measurement. Note that in the course of the optimization process, the remaining budget decreases, so $\tau^{2}\left(T_{n+1}\right)$ [respectively, $\tau^{2}\left(t_{i} \rightarrow t_{i}+T_{n+1}\right)$ ] increases with $n$.

Then, we propose to set $\tau_{n+1}^{2}=\tau^{2}\left(T_{n+1}\right)$ [respectively, $\left.\tau^{2}\left(t_{i} \rightarrow t_{i}+T_{n+1}\right)\right]$ for the EQI calculation, meaning that the EQI will measure the potential improvement if all the remaining budget would be attributed to the next observation. Of course, the actual budget $t_{n+1}$ for the next observation may be a lot smaller than $T_{n+1}$, so the optimization does not stop after one step. With this setting, the new experiment is chosen knowing that even if all the budget was used for a single observation, its noise variance would not decrease below a certain value.

Consequently, the EQI will behave differently at the beginning and at the end of the optimization. When the budget is high, EQI will tendencially be higher in unexplored regions, since it is where accurate measurements are likely to be most efficient (the EQI will actually be almost similar to a classical EI). At the end of the optimization, however, when the remaining time is small, the EQI will be small in unexplored regions since even if the actual function is low, there is not enough computational time to obtain a lower quantile than the current best one. In that case, the EQI will be highest close to or at the current best point(s) and favor local search.

\section{ALLOCATION OF RESOURCES}

This section proposes two algorithmic schemes based on EQI. First, the baseline approach is described, where a fixed time is allocated at each iteration; then, a strategy is proposed to take advantage of the response convergence monitoring to dynamically adapt the budget to each measurement.

\subsection{Constant Allocation}

First, we assume that the computational budget $T_{0}$ can be divided in elementary time steps $t_{e}$ so that $T_{0}=N \times t_{e}$. An elementary step can correspond to a given number of solver iterations for partial convergence, or to a number of drawings for stochastic simulators. An algorithm with constant allocation will then proceed to attribute each of the $N$ elementary time steps to either generate new measurements or improve accuracy on existing ones.

At step $n$, a budget $n \times t_{e}<T_{0}$ has already been spent on the measurements, so $T_{n+1}=T_{0}-n \times t_{e}$. The EQI criterion is maximized over $D$ with $\tau_{n+1}^{2}=\tau^{2}\left(T_{n+1}\right)$ at unsampled locations and $\tau_{n+1}^{2}=\tau^{2}\left(t_{i} \rightarrow t_{i}+T_{n+1}\right)$ at $\mathbf{x}^{i}(1 \leq i \leq n)$. Once the new point $\mathbf{x}^{n+1}$ is chosen and the measurement is made, the kriging model has to be updated, by either adding $\mathbf{x}^{n+1}$ to $\mathbf{X}^{n}$ if $\mathbf{x}^{n+1} \notin$ $\mathbf{X}^{n}$ or by replacing the previous values of $\tilde{y}_{i}\left[b_{i}\right]$ and $\tau_{i}^{2}\left[b_{i}\right]$ by $\tilde{y}_{i}\left[b_{i}+1\right]$ and $\tau_{i}^{2}\left[b_{i}+1\right]$, respectively, in the kriging equations.

The trade-off between precision and number of measurements is here determined by EQI, depending if it is maximum at a sampled or an unsampled design. Taking the finite budget into account affects the trade-off, since with large budget it is more exploratory, hence likely to be maximum at unsampled points, while with small budget it is more likely to be maximal at existing points (see Figure 2, EQI with $\beta=0.9$ and $\tau^{2}=0.1$ or $0.01)$.

Note that this procedure allows the (closely related) problem of optimization of a homogeneously noisy function to be addressed, considering that each observation requires a constant time $t_{e}$ and has a constant noise variance $v^{2}$. At each optimization step, the user has the option of either sampling at a new location or duplicating an existing measurement, so allocating all the remaining budget to a measurement means performing $N-n$ replications at this point, hence leading to the situation described in Section 2.3 [Equation (1)]. In that case, it is straightforward to get $\tau^{2}\left(t_{i} \rightarrow t_{i}+T_{n+1}\right)=\frac{v^{2}}{N-n}=\tau^{2}\left(T_{n+1}\right)$, so the criterion is written similarly at sampled and unsampled locations. Thus, in this framework, the procedure simplifies to maximizing at each step $\operatorname{EQI}_{n}\left(., \frac{v^{2}}{N-n}\right)$.

\subsection{Online Allocation}

The constant allocation strategy of the previous section performs $N-n_{0}$ EGO iterations, and each requires the running of an inner optimization loop for the maximization of the EQI, which can be very time consuming. Hence, the elementary time step $t_{e}$ must be chosen to be large enough to limit the number of EQI optimizations (otherwise choosing the next measurement could take more time than performing it!). Typically, with partial convergence or stochastic simulators, $t_{e}$ must be chosen to be much larger than a single solver iteration or drawing, respectively. This limitation can greatly hinder the flexibility and potential of tunable precision, since it reduces the possibilities of a quasi-continuum of fidelities to a few discrete precision levels. 
Table 1. EQI algorithm with online resource allocation

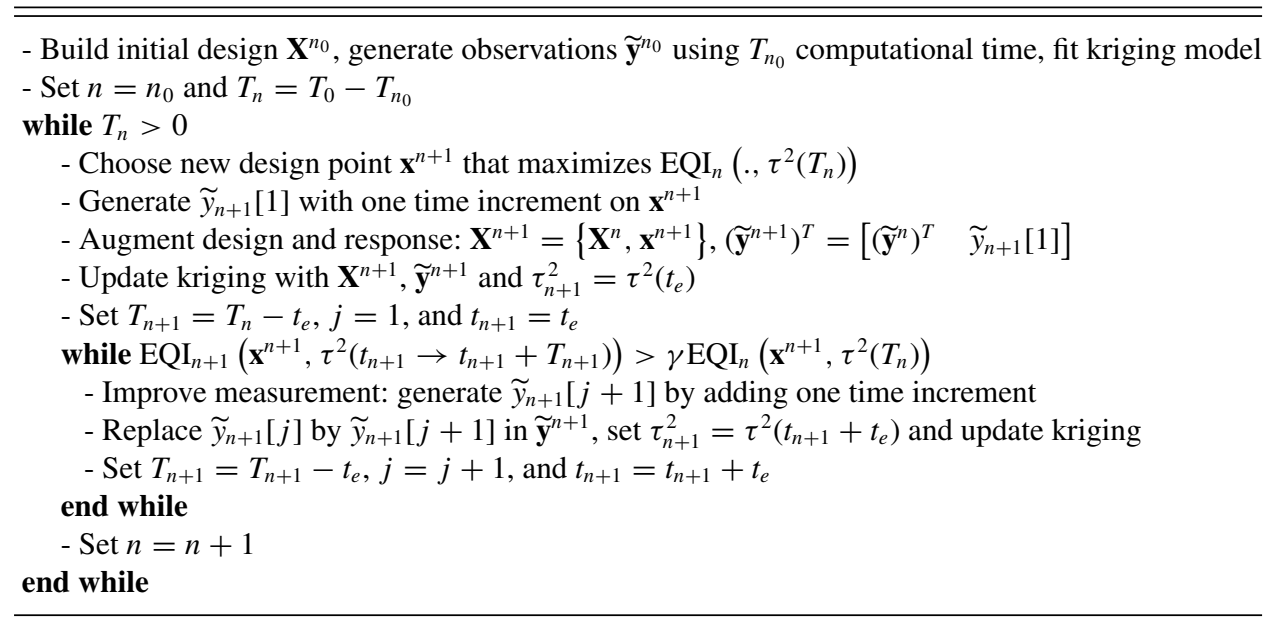

Here, we propose a heuristic for dynamically choosing the computational resource given to an experiment. A simple way to do so is to monitor the evolution of the EQI at the current observation point. Indeed, instead of maximizing the EQI after each $t_{e}$ is spent, we will choose an observation point and allocate several time steps on it until a criterion is met. As for the constant allocation case, the EQI is updated after each step, by replacing the previous values of response and noise $\tilde{y}_{i}\left[b_{i}\right]$ and $\tau_{i}^{2}\left[b_{i}\right]$ by $\tilde{y}_{i}\left[b_{i}+1\right]$ and $\tau_{i}^{2}\left[b_{i}+1\right]$, respectively, in the kriging equations, and by replacing the future noise level $\tau^{2}\left(t_{i} \rightarrow t_{i}+T_{n+1}\right)$ by $\tau^{2}\left(t_{i}+t_{e} \rightarrow t_{i}+T_{n+1}-t_{e}\right)$.

By construction, the updated EQI tends to decrease when computation time is added, since (a) the kriging uncertainty reduces at the observation point and (b) EQI decreases when $\tau^{2}\left(t_{i} \rightarrow t_{i}+T_{n+1}\right)$ increases. However, if the measurement converges to a good (small) value, EQI can increase temporarily. Conversely, if the measurement converges to a high value, EQI decreases faster. Hence, we can define a ("point switching") stopping criterion for resource allocation based on EQI. If the EQI decreases below a certain value, carrying on the calculations is not likely to help the optimization, so the observation process should stop and another point should be chosen. Here, we propose the interruption of a measurement and search for a new point when the current value of the EQI is less than a proportion of the initial EQI value (i.e., the value of EQI when starting the measurement process at that point), for instance $50 \%$.

The sequence of this new procedure is as follows: first, find $\mathbf{x}^{n+1}=\arg \max _{\mathbf{x} \in D}\left(\mathrm{EQI}(\mathbf{x}), \tau^{2}\left(T_{n+1}\right)\right)$, store the corresponding value $\left.\mathrm{EQI}\left(\mathbf{x}^{n+1}\right), \tau^{2}\left(T_{n+1}\right)\right)$ as reference, and then invest elementary measurements at this point until the EQI with updated data falls under a given proportion $\gamma \in] 0,1[$ of the reference value. The operation of choosing the most promising point is then started again and so on until the total computational budget has been spent. Note that the final number of measurements and EQI maximizations are not determined beforehand but adapts automatically to the budget and resource distribution, and may be a lot smaller than the number of steps $N$ (especially with small $\gamma)$. The algorithm is presented in pseudo-code form in Table 1 . For conciseness, this algorithm does not consider the case where $\mathbf{x}^{n+1} \in \mathbf{X}^{n}$, which requires different treatement, as in Section 6.1. In the examples in Section 7, $\mathbf{x}^{n+1} \in \mathbf{X}^{n}$ is considered.

\section{EXPERIMENTS}

\subsection{Comparison to the Augmented Expected Improvement Procedure}

The strategy proposed in Section 6.2 is compared with the AEI method as proposed in Huang et al. (2006) for the optimization of homogeneously noisy experiments, which has already been found to be very competitive compared with other local or global optimizers such as the revised simplex search (Humphrey and Wilson 2000) or DIRECT (Gablonsky and Kelley 2001). Both EQI and AEI heuristics are compared with the classical EI, using a noisy kriging model (as in Section 2.4) and with the minimal value of the observations replaced by the minimum of the kriging mean at the observations, which can be considered as the baseline approach. As test problems, we employed two analytical benchmark problems, the $d=6$ dimensional Hartman function (Dixon and Szego 1978) and the $d=5$ dimensional Ackley function (Ackley 1987).

Hartman:

$$
y(\mathbf{x})=\frac{-1}{1.94}\left[2.58+\sum_{i=1}^{4} C_{i} \exp \left(-\sum_{j=1}^{6} a_{j i}\left(x_{j}-p_{j i}\right)^{2}\right)\right]
$$

with: $\mathbf{C}=\left[\begin{array}{l}1.0 \\ 1.2 \\ 3.0 \\ 3.2\end{array}\right], \mathbf{a}=\left[\begin{array}{cccc}10.00 & 0.05 & 3.00 & 17.00 \\ 3.00 & 10.00 & 3.50 & 8.00 \\ 17.00 & 17.00 & 1.70 & 0.05 \\ 3.50 & 0.10 & 10.00 & 10.00 \\ 1.70 & 8.00 & 17.00 & 0.10 \\ 8.00 & 14.00 & 8.00 & 14.00\end{array}\right]$

$$
\mathbf{p}=\left[\begin{array}{llll}
0.1312 & 0.2329 & 0.2348 & 0.4047 \\
0.1696 & 0.4135 & 0.1451 & 0.8828 \\
0.5569 & 0.8307 & 0.3522 & 0.8732 \\
0.0124 & 0.3736 & 0.2883 & 0.5743 \\
0.8283 & 0.1004 & 0.3047 & 0.1091 \\
0.5886 & 0.9991 & 0.6650 & 0.0381
\end{array}\right]
$$


Table 2. Summary of the test problem configurations

\begin{tabular}{lcll}
\hline \hline Function & Initial design size $n_{0}$ & Budget $T_{0}$ & $\tau$ \\
\hline Ackley & 25 points & 500 steps & 0.05 \\
Ackley & 50 points & 1000 steps & 0.2 \\
Hartman & 60 points & 1200 steps & 0.2 \\
\hline
\end{tabular}

$$
\text { Ackley: } \begin{aligned}
y(\mathbf{x})= & -20 \exp \left(-0.2 \sqrt{\frac{1}{d} \sum_{i=1}^{d} x_{i}^{2}}\right) \\
& -\exp \left(\frac{1}{d} \sum_{i=1}^{d} \cos \left(2 \pi x_{i}\right)\right)+20+\exp (1)
\end{aligned}
$$

Both functions are normalized so their design region $D$ is $[0,1]^{d}$ and their standard deviation (SD) is 1.0 over $D$. Their minima are zero for Ackley and -1.94 for Hartman. Gaussian noise $\epsilon \sim \mathcal{N}\left(0,10 \tau^{2}\right)$ is added to the analytical functions. An ordinary kriging model (constant trend) with Matern $5 / 2$ anisotropic covariance function is used for both functions.

To model a tunable fidelity framework while allowing a fair comparison between methods, each noisy measurement $\tilde{y}_{i}$ is taken as the average of several function evaluations as described in Section 2.3. For the AEI procedure, which is designed for homoscedastic noise, 10 time steps are used for each observation, so the noise variance is $\tau^{2}$. For the EQI procedure, the noise variance potentially varies between $10 \tau^{2}$ and $10 \tau^{2} / T_{0}$.

For both methods, the initial design sets are chosen as Latin hypercube sampling (LHS) designs with maximin criterion, and are generated using 10 time steps for each observation. The total optimization budget is chosen to be equal to two times the budget needed to generate the initial design set. Two versions of EQI are tested, with $\beta=0.5$ (decision based on kriging mean only) and with $\beta=0.9$; the criteria are referred to as EQI.50 and EQI.90, respectively.

Several budgets and noise levels are tested. The different configurations are summarized in Table 2. The noise level $\tau$ can be compared to objective function SD, which is one for both functions. With $\tau=0.2$, the optimization problem can be considered as very noisy. The total budget is deliberately chosen to be very small since it may correspond to typical situations in real-life applications.

For each configuration, 40 initial designs and observations are generated to account for randomness in the LHS designs and the observations. The kriging parameters are estimated only at the initial step, using the $\mathrm{R}$ package DiceKriging (Roustant, Ginsbourger, and Deville 2011), so all the methods use the same models. Sequential parameter reestimation is not done here so the algorithms can be compared in terms of prediction variance or quantile (otherwise, the model with larger range and lower process variance would typically have a lower prediction variance, regardless of the design sets).

The current minimizer for AEI or EQI.90 is $\mathbf{x}^{*}=$ $\arg \min _{1 \leq i \leq n} q_{n}\left(\mathbf{x}_{i}\right)$, and $\arg \min _{1 \leq i \leq n} m_{n}\left(\mathbf{x}_{i}\right)$ for EQI.50 or EI. The optimization performances are compared based on $y\left(\mathbf{x}^{*}\right)$ (actual value at $\mathbf{x}^{*}$ ) and $s_{n}\left(\mathbf{x}^{*}\right)$ (kriging SD), which are represented using boxplots on Figure 3 .
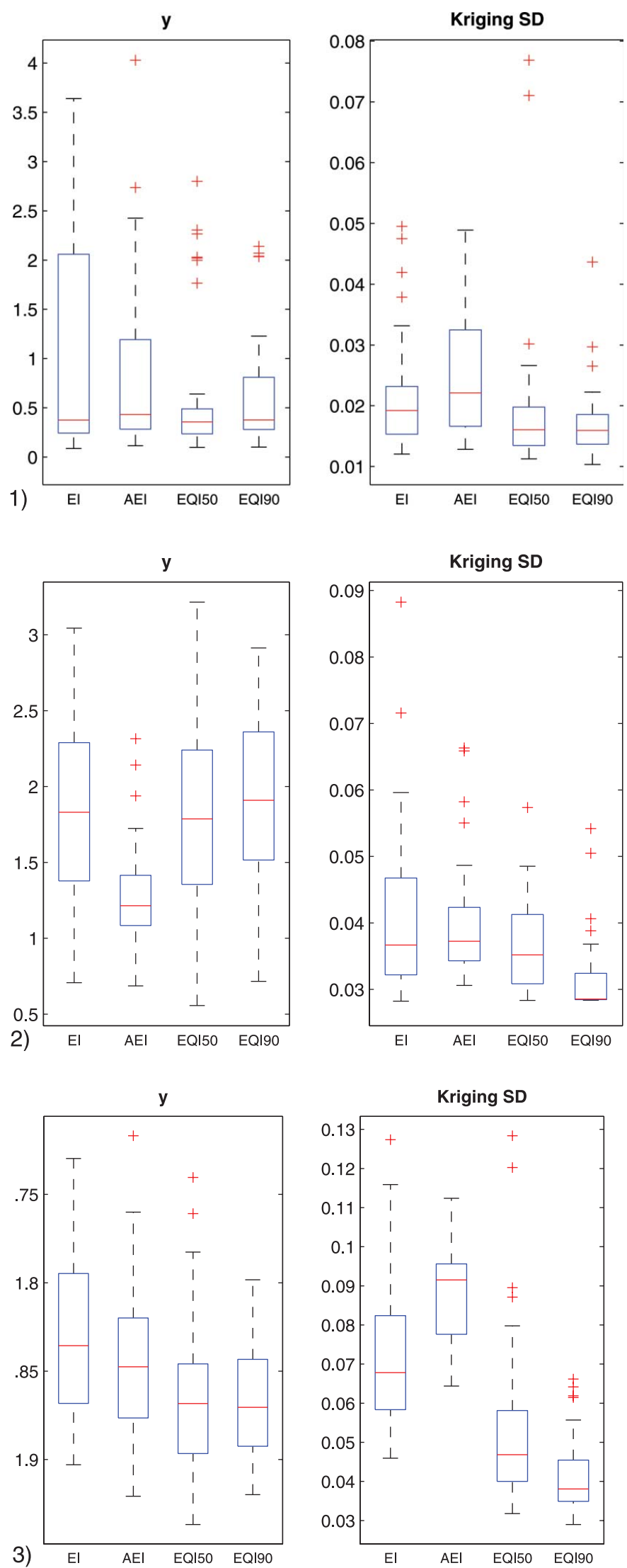

Figure 3. Boxplots of actual value $(y)$ and kriging SD $\left(s_{n}\right)$ at $\mathbf{x}^{*}$ for the different methods on (1) the Ackley function with $\tau=0.05$ and 500 steps, (2) the Ackley function with $\tau=0.2$ and 1000 steps, and (3) the Hartman function with $\tau=0.2$ and 1200 steps. The online version of this figure is in color. 
Table 3. Computational time allocation during optimization

\begin{tabular}{|c|c|c|c|c|c|c|c|c|}
\hline \multirow[b]{2}{*}{ Configuration (Function, initial design, budget, $\tau$ ) } & \multicolumn{4}{|c|}{ Number of distinct measurements } & \multicolumn{4}{|c|}{ Time steps at $\mathbf{x}^{*}$} \\
\hline & EQI.50 & EQI.90 & AEI & EI & EQI.50 & EQI.90 & AEI & EI \\
\hline Ackley, 50 points, 1000 steps, 0.2 & 72 & 63 & 87 & 98 & 21 & 64 & 15 & 11 \\
\hline Hartman, 60 points, 1200 steps, 0.2 & 130 & 118 & 117 & 103 & 5 & 8 & 23 & 38 \\
\hline
\end{tabular}

For the Ackley function, with $\tau=0.05$, EQI outperforms AEI in terms of actual value and kriging uncertainty at $\mathbf{x}^{*}$. The choice of $\beta=0.5$ provides the best results. With $\tau=0.2$, AEI provides the best results in terms of optimization $\left(y\left(\mathbf{x}^{*}\right)\right)$. However, $s_{n}\left(\mathbf{x}^{*}\right)$ is significantly lower for EQI.90 than for the other methods, which illustrates the tendency of this method to reduce uncertainty at the expense of exploration.

For the Hartman function, EQI slightly outperforms the two other methods in terms of $y\left(\mathbf{x}^{*}\right)$. In terms of kriging uncertainty, EI and AEI are clearly less efficient than EQI. The difference between the strategies $\beta=0.5$ and $\beta=0.9$ appear clearly; indeed, with $\beta=0.5$, the choice of the best design is made on the kriging mean only; on the other hand, with $\beta=0.9$, observations with high uncertainty are penalized so $s_{n}\left(\mathbf{x}^{*}\right)$ is always small.

Table 3 shows the average number of distinct measurements and the average number of time steps at $\mathbf{x}^{*}$. Even though EI and AEI use uniform allocation, their values are not constant because some measurements are repeated (i.e., criteria are maximal at existing measurements during optimization). For instance, the first row and last column of the table indicates that for EI, there is on average four repeated measurements at $\mathbf{x}^{*}$.

For the small budget and small noise on the Ackley function, the online allocation of Section 6.2 resulted with more measurements than for AEI and EI. Here, online allocation was used to improve exploration by having more measurement locations and resulted in better performance in terms of $y\left(\mathbf{x}^{*}\right)$ (see Figure 3$)$. On the contrary, for the large budget and large noise, it resulted in accurate measurements at $\mathbf{x}^{*}$ to the detriment of exploration. For the Hartman function, the number of measurements is almost equivalent for all methods.

It is interesting to note that for the Ackley function with $\tau=0.05$ and the Hartman function, the average number of time steps at $\mathbf{x}^{*}$ is smaller for EQI than for EI and AEI but $s_{n}\left(\mathbf{x}^{*}\right)$ is also smaller (see Figure 3), which is counter intuitive. For EQI, the small $s_{n}\left(\mathbf{x}^{*}\right)$ is obtained because the measurements form a cluster around $\mathbf{x}^{*}$.

\subsection{Application to a 2D Benchmark From Nuclear Criticality Safety Assessments}

In this section, the optimization algorithm is applied to the problem of safety assessment of a nuclear system involving fissile materials. The benchmark system used is an interim storage of dry $\mathrm{PuO}_{2}$ powder into a regular array of storage tubes. The criticality safety of this system is evaluated through the neutron multiplication factor (called $k$-effective or $k_{\text {eff }}$ ), which models the nuclear chain reaction trend: $k_{\text {eff }}>1$ implies an increasing neutron production leading to an uncontrolled chain reaction, and $k_{\text {eff }}<1$ is the safety state required for fuel storage.

The neutron multiplication factor depends on many parameters such as the composition of fissile materials, operation conditions, geometry, etc. For a given set of parameters, the value of $k_{\text {eff }}$ can be evaluated using the MORET stochastic simulator (Fernex et al. 2005), which is based on Markov chain Monte Carlo (MCMC) simulation techniques. The precision of the evaluation depends on the amount of simulated particles (neutrons), which is tunable by the user.

When assessing the safety of a system, one has to ensure that, given a set of admissible values $D$ for the parameters $\mathbf{x}$, there are no physical conditions under which the $k_{\text {eff }}$ can reach the critical value of one. The search for the worst combination of parameters $\mathbf{x}$ defines a noisy optimization problem which is often challenging in practice, due to the high computational expense of the simulator. An efficient resolution technique for this problem is particularly crucial since this optimization may be done numerous times.

In this article, we focus on the maximization of $k_{\text {eff }}$ with respect to two parameters, the other possible inputs being fixed to their most penalizing values (based on expert knowledge):

(a) $d . p u o 2$, the powder density, with original range $[0.5,4]$ $\mathrm{g} \cdot \mathrm{cm}^{-3}$, rescaled to $[0,1]$,

(b) d.water, the density of water between storage tubes, with range $[0,1]$, which accounts for the possible flooding of the storage (leading to an interstitial moderation of the neutrons interacting from a storage tube to another one).

Hence, to agree with previous notations, we set $\mathbf{x}=$ (d.puo2, d.water $)$ and $y(\mathbf{x})=-k_{\mathrm{eff}}(\mathbf{x})$. Simulation time is assumed to be proportional to the number of neutrons simulated (the entry cost of a new simulation being neglected). Since the simulator is based on Monte Carlo, the variance of the $k_{\text {eff }}$ estimate is exactly inversely proportional to the number of neutrons. The variance slightly varies with input parameters, but this dependence can be considered negligible here.

For practical considerations, the optimization space $D$ is discretized in a $75 \times 75$ grid, and for each new measurement, the EQI maximization is performed by an exhaustive search on the grid. The incremental time step $t_{e}$ is defined by the simulation of 4000 particles, which takes about half a minute on a $3 \mathrm{GHz}$ CPU. The response noise SD can take values between $5.67 \times 10^{-2}$ (one time step) and $4.01 \times 10^{-3}$ (200 time steps). 

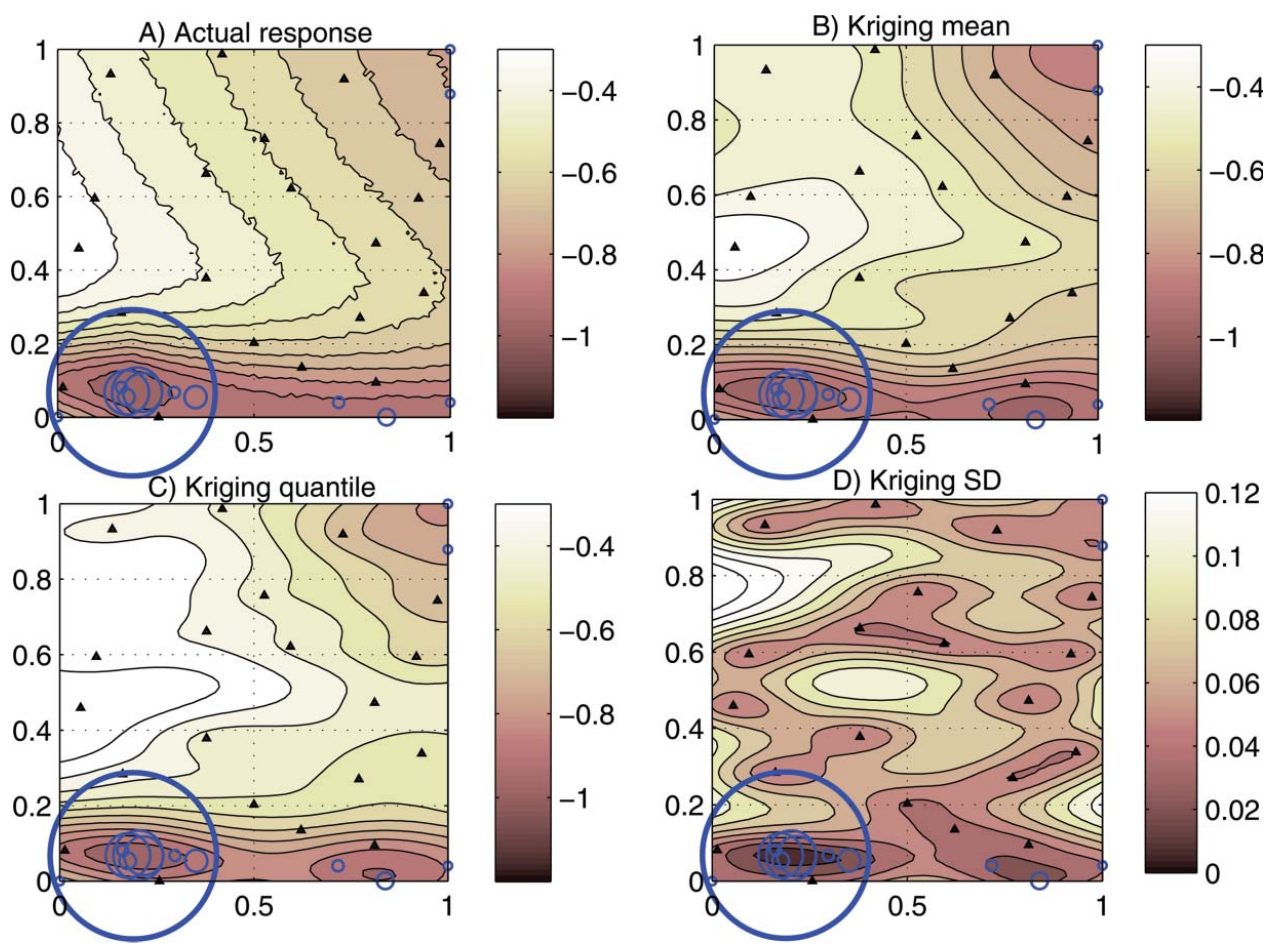

Figure 4. Optimization results for a $T=100$ budget. The $x$-axes correspond to $d$.puo 2 and the $y$-axes to d.water. Triangles represent the inital measurements and circles the added ones; the markers are proportional to computational time. The online version of this figure is in color.

To evaluate the efficiency of our algorithm, all 5625 points of the grid have been evaluated with high precision, which gives an accurate estimation of the shape [represented in Figure 4(A)], minimal value, and minimizer $\mathbf{x}^{*}$ of the function. With this accurate dataset, we find that $\mathbf{x}^{*}=[0.1892,0.0811]$ and $f\left(\mathbf{x}^{*}\right)=$ -0.9847 .

The $k_{\text {eff }}$ range is approximately [0.3, 1.0], so with one time step, the measurement $95 \%$ confidence interval length is $4 \times 0.0567=0.226$, which is about $30 \%$ of the response range. With 200 time steps, this length is $2 \%$ of the range. We consider a computational budget of $T_{0}=100$, which corresponds to a single observation with SD of $5.7 \times 10^{-3}$. This can be considered as a very small budget regarding the problem complexity. The initial design consists of a 20-point random design (with optimized maximin distance), with one time step used for each measurement (so $20 \%$ of the budget is allocated to the initial design).

The kriging fit is made using the $\mathrm{R}$ package DiceKriging (Roustant, Ginsbourger, and Deville 2011). The chosen model has a constant trend (ordinary kriging) and Matern $5 / 2$ anisotropic covariance function. The covariance parameters are reestimated after each new observation.

Figure 4 shows the contour lines of the actual response, the final kriging (mean, SD, and 90th percentile), and measurements. During optimization, 14 measurements have been added with time steps varying from 1 to 36 . The best design point found is $\mathbf{x}^{*}=[0.189,0.068]$, has a kriging SD of 0.0071 and is almost equal to the actual minimizer. Here, approximately one third of the computational budget is allocated to $\tilde{y}\left(\mathbf{x}^{*}\right)$. The final kriging [Figure 4(B) and 4(D)] is relatively accurate, even though the $\mathrm{SD}$ remains high in all the regions with high response values (top left quadrant). In the region of the optimum, the kriging 90th percentile [Figure $4(\mathrm{C})$ ] is almost equal to the actual function.

\section{CONCLUSION AND PERSPECTIVES}

In this article, we have proposed a quantile-based EI for the optimization of noisy back-box simulators [available in the $\mathrm{R}$ package DiceOptim Ginsbourger, Picheny, Roustant, with contributions by C. Chevalier and Wagner 2012)]. This criterion allows an elegant treatment of heterogeneous noise and takes into account the noise level of the candidate measurements. In the context of simulators with tunable fidelity, we proposed an online procedure for an adapted distribution of the computational effort. One of the advantages of such procedure is that it guards against the allocation of too much time to poor designs, and focuses more effort on the best ones. Another remarkable property of this algorithm is that, unlike EGO, it takes into account the limited computational budget. Indeed, the algorithm is more exploratory when there is much budget left and favors a more local search when resources are scarce. The online allocation optimization algorithm was first compared with existing methods on two analytical benchmark functions and was found to be very competitive. Finally, it was applied to an original application in nuclear criticality safety, the Monte Carlo criticality simulator MORET5. The algorithm showed promising results, using coarse measurements for exploration and accurate measurements at best designs. Future work may include a deeper comparison of the EQI to other criteria for point selection on an extended benchmark of test functions, analysis of the effect of online allocation compared with a uniform allocation 
strategy, and an adaptation of the algorithm in the case of correlated errors.

\section{APPENDIX: ONE-STEP AHEAD CONDITIONAL DISTRIBUTIONS OF THE MEAN, VARIANCE, AND QUANTILE PROCESSES}

Let $\mathbf{x}^{n+1}$ be the point to be visited at the $(n+1)$ th step, $\tau_{n+1}^{2}$ and $\tilde{Y}_{n+1}=Y\left(\mathbf{x}^{n+1}\right)+\varepsilon_{n+1}$ the corresponding noise variance and noisy response, respectively. We will now discuss the properties of the kriging mean and variance at step $n+1$ seen from step $n$.

Let $M_{n+1}(\mathbf{x}):=\mathbb{E}\left[Y(\mathbf{x}) \mid \widetilde{A_{n}}, \widetilde{Y}_{n+1}\right]$ be the kriging mean function at the $(n+1)$ th step and $S_{n+1}^{2}(\mathbf{x}):=\operatorname{var}\left[Y(\mathbf{x}) \mid \widetilde{A}_{n}, \widetilde{Y}_{n+1}\right]$ the corresponding conditional variance.

Seen from step $n$, both of them are ex ante random processes since they are depending on the not yet observed measurement $\widetilde{Y}_{n+1}$. We will now prove that they are in fact GPs $\mid \widetilde{A_{n}}$, as well as the associated quantile $Q_{n+1}(\mathbf{x})=M_{n+1}(\mathbf{x})+\Phi^{-1}(\beta) S_{n+1}(\mathbf{x})$.

The key results are that the kriging predictor is linear in the observations and that the kriging variance is independent of them, as can be seen from (3) and (4). Writing

$M_{n+1}(\mathbf{x})=\left(\sum_{j=1}^{n} \lambda_{n+1, j}(\mathbf{x}) \widetilde{Y}_{j}\right)+\lambda_{n+1, n+1}(\mathbf{x})\left(Y\left(\mathbf{x}^{n+1}\right)+\varepsilon_{n+1}\right)$,

where

$$
\begin{aligned}
& \left(\lambda_{n+1, .}(\mathbf{x})\right) \\
& :=\left(\mathbf{k}_{n+1}(\mathbf{x})^{T}+\frac{\left(1-\mathbf{k}_{n+1}(\mathbf{x})^{T}\left(K_{n+1}+\Delta_{n+1}\right)^{-1} \mathbf{1}_{n+1}\right)}{\mathbf{1}_{n+1}^{T}\left(K_{n+1}+\Delta_{n+1}\right)^{-1} \mathbf{1}_{n+1}} \mathbf{1}_{n+1}^{T}\right) \\
& \quad \times\left(K_{n+1}+\Delta_{n+1}\right)^{-1},
\end{aligned}
$$

it appears that $M_{n+1}$ is a GP $\mid \widetilde{A_{n}}$, with the following conditional mean and covariance kernel:

$$
\begin{aligned}
\mathbb{E} & {\left[M_{n+1}(\mathbf{x}) \mid \widetilde{A_{n}}\right] } \\
& =\sum_{j=1}^{n} \lambda_{n+1, j}(\mathbf{x}) \tilde{y}_{i}+\lambda_{n+1, n+1}(\mathbf{x}) m_{n}(\mathbf{x})
\end{aligned}
$$

and

$$
\begin{aligned}
& \operatorname{cov}\left[M_{n+1}(\mathbf{x}), M_{n+1}\left(\mathbf{x}^{\prime}\right) \mid \widetilde{A_{n}}\right] \\
& \quad=\lambda_{n+1, n+1}(\mathbf{x}) \lambda_{n+1, n+1}\left(\mathbf{x}^{\prime}\right)\left(s_{n}^{2}\left(\mathbf{x}^{n+1}\right)+\tau_{n+1}^{2}\right) .
\end{aligned}
$$

Using the fact that $Q_{n+1}(\mathbf{x})=M_{n+1}(\mathbf{x})+\Phi^{-1}(\beta) S_{n+1}(\mathbf{x})$, we observe that seen from the $n$th step, $Q_{n+1}($.) is a GP as sum of a GP and a deterministic process conditional on $\widetilde{A_{n}}$. Finally,

$$
\begin{aligned}
\mathbb{E} & {\left[Q_{n+1}(\mathbf{x}) \mid \widetilde{A_{n}}\right] } \\
& =\sum_{j=1}^{n} \lambda_{n+1, j}(\mathbf{x}) \tilde{y}_{i}+\lambda_{n+1, n+1}(\mathbf{x}) m_{n}(\mathbf{x})+\Phi^{-1}(\beta) s_{n+1}(\mathbf{x}),
\end{aligned}
$$

$$
\begin{aligned}
& \operatorname{cov}\left[Q_{n+1}(\mathbf{x}), Q_{n+1}\left(\mathbf{x}^{\prime}\right) \mid \widetilde{A_{n}}\right] \\
& \quad=\lambda_{n+1, n+1}(\mathbf{x}) \lambda_{n+1, n+1}\left(\mathbf{x}^{\prime}\right)\left(s_{n}^{2}\left(\mathbf{x}^{n+1}\right)+\tau_{n+1}^{2}\right),
\end{aligned}
$$

and the values used in the EQI are

$$
m_{Q_{n+1}}=\mathbb{E}\left[Q_{n+1}\left(\mathbf{x}^{n+1}\right) \mid \widetilde{A_{n}}\right]
$$

$$
\begin{aligned}
s_{Q_{n+1}}^{2} & =\operatorname{var}\left[Q_{n+1}\left(\mathbf{x}^{n+1}\right) \mid \widetilde{A_{n}}\right] \\
& =\left(\lambda_{n+1, n+1}\left(\mathbf{x}^{n+1}\right)\right)^{2}\left(s_{n}^{2}\left(\mathbf{x}^{n+1}\right)+\tau_{n+1}^{2}\right) .
\end{aligned}
$$

Now, we show that we can write $m_{Q_{n+1}}$ and $s_{Q_{n+1}}^{2}$ as a function of the kriging values at step $n$. Indeed, as can be shown in the fashion of Emery (2009), the mean, variance, and weights of the kriging updated with an additional measurement $\tilde{y}_{n+1}$ performed at $\mathbf{x}_{n+1}$ with variance $\tau_{n+1}^{2}$ are equal to

$$
\begin{aligned}
& m_{n+1}(\mathbf{x})=m_{n}(\mathbf{x})+\frac{c_{n}\left(\mathbf{x}, \mathbf{x}^{n+1}\right)}{s_{n}^{2}\left(\mathbf{x}^{n+1}\right)+\tau_{n+1}^{2}}\left(\tilde{y}_{n+1}-m_{n}\left(\mathbf{x}^{n+1}\right)\right) \\
& s_{n+1}^{2}(\mathbf{x})=s_{n}^{2}(\mathbf{x})-\frac{c_{n}\left(\mathbf{x}, \mathbf{x}^{n+1}\right)^{2}}{s_{n}^{2}\left(\mathbf{x}^{n+1}\right)+\tau_{n+1}^{2}} \\
& \lambda_{n+1}(\mathbf{x})=\left[\begin{array}{c}
\lambda_{n}(\mathbf{x})-\frac{c_{n}\left(\mathbf{x}, \mathbf{x}^{n+1}\right)}{s_{n}^{2}\left(\mathbf{x}^{n+1}\right)+\tau_{n+1}^{2}} \lambda_{n}\left(\mathbf{x}^{n+1}\right) \\
\frac{c_{n}\left(\mathbf{x}, \mathbf{x}^{n+1}\right)}{s_{n}^{2}\left(\mathbf{x}^{n+1}\right)+\tau_{n+1}^{2}}
\end{array}\right],
\end{aligned}
$$

where $c_{n}$ is the kriging covariance, equal (for ordinary kriging) to

$$
\begin{aligned}
c_{n}\left(\mathbf{x}, \mathbf{x}^{\prime}\right)= & k\left(\mathbf{x}, \mathbf{x}^{\prime}\right)-\mathbf{k}_{n}(\mathbf{x})^{T} \mathbf{K}_{n}^{-1} \mathbf{k}_{n}\left(\mathbf{x}^{\prime}\right) \\
& +\frac{\left(1-\mathbf{k}_{n}(\mathbf{x}) \mathbf{K}_{n}^{-1} \mathbf{1}_{n}\right)\left(1-\mathbf{1}_{n}^{T} \mathbf{K}_{n}^{-1} \mathbf{k}_{n}\left(\mathbf{x}^{\prime}\right)\right)}{\mathbf{1}_{n}^{T} \mathbf{K}_{n}^{-1} \mathbf{1}_{n}}
\end{aligned}
$$

Then, noting that $c_{n}(\mathbf{x}, \mathbf{x})=s_{n}^{2}(\mathbf{x})$ and that setting $\tilde{y}^{n+1}=$ $m_{n}\left(\mathbf{x}^{n+1}\right)$ implies $m_{n+1}(\mathbf{x})=m_{n}(\mathbf{x}), \quad$ (A.6) and (A.7) simplify to

$$
\begin{aligned}
m_{Q_{n+1}} & =m_{n}\left(\mathbf{x}^{n+1}\right)+\Phi^{-1}(\beta) \sqrt{\frac{\tau_{n+1}^{2} \times s_{n}^{2}\left(\mathbf{x}^{n+1}\right)}{s_{n}^{2}\left(\mathbf{x}^{n+1}\right)+\tau_{n+1}^{2}}} \\
s_{Q_{n+1}}^{2} & =\frac{\left[s_{n}^{2}\left(\mathbf{x}^{n+1}\right)\right]^{2}}{s_{n}^{2}\left(\mathbf{x}^{n+1}\right)+\tau_{n+1}^{2}}
\end{aligned}
$$

\section{SUPPLEMENTARY MATERIALS}

Equivalent measurement for two noisy observations at the same point: Proof of equivalence between including two independent noisy observations at the same point in the kriging equations and including a single equivalent observation. (pdf file)

\section{ACKNOWLEDGMENTS}

This work was partially supported by French National Research Agency (ANR) through COSINUS program (project OMD2 ANR-08-COSI-007).

[Received October 2010. Revised January 2012.]

\section{REFERENCES}

Ackley, D. (1987), A Connectionist Machine for Genetic Hillclimbing, Hingham, MA: Kluwer. [8]

Dixon, L., and Szego, G. (1978), Towards Global Optimisation 2, Amsterdam: North-Holland. [8]

Emery, X. (2009), "The Kriging Update Equations and Their Application to the Selection of Neighboring Data," Computational Geosciences, 13, 269 280. [12] 
Fedorov, V., and Hackl, P. (1997), Model-Oriented Design of Experiments, Berlin: Springer. [7]

Fernex, F., Heulers, L., Jacquet, O., Miss, J., and Richet, Y. (2005), "The MORET 4B Monte Carlo Code-New Features to Treat Complex Criticality Systems," in $M \& C$ International Conference on Mathematics and Computation Supercomputing, Reactor Physics and Nuclear and Biological Application, Avignon, France. [10]

Forrester, A., Bressloff, N., and Keane, A. (2006), "Optimization Using Surrogate Models and Partially Converged Computational Fluid Dynamics Simulations," Proceedings of the Royal Society A, 462 (2071), 21772204. [3]

Forrester, A., Keane, A., and Bressloff, N. (2006), "Design and Analysis of 'Noisy' Computer Experiments," AIAA Journal, 44, 2331-2339. [2,3]

Forrester, A., Sóbester, A., and Keane, A. (2007), "Multi-Fidelity Optimization via Surrogate Modelling," Proceedings of the Royal Society A: Mathematical, Physical and Engineering Science, 463 (2088), 3251-3269. [2,3]

Gablonsky, J., and Kelley, C. (2001), "A Locally-Biased Form of the DIRECT Algorithm," Journal of Global Optimization, 21, 27-37. [8]

Gano, S., Renaud, J., Martin, J., and Simpson, T. (2006), "Update Strategies for Kriging Models Used in Variable Fidelity Optimization," Structural and Multidisciplinary Optimization, 32, 287-298. [2]

Ginsbourger, D., and Le Riche, R. (2010), "Towards GP-Based Optimization With Finite Time Horizon," in mODa 9 Advances in Model-Oriented Design and Analysis, eds. A. Giovagnoli, A. C. Atkinson, B. Torsney, and C. May, Contributions to Statistics Physica-Verlag HD, Heidelberg: Springer, pp. 89-96. [7]

Ginsbourger, D., Picheny, V., Roustant, O., with contributions by C. Chevalier, and Wagner, T. (2012), DiceOptim: Kriging-Based Optimization for Computer Experiments, R package version 1.3, Vienna: R Foundation for Statistical Computing. [11]

Gramacy, R., and Lee, H. (2012), "Cases for the Nugget in Modeling Computer Experiments," Statistics and Computing, 22, 713-722. [3] (2010b), "Optimization Under Unknown Constraints," in Bayesian Statistics 9, eds. J. M. Bernardo, M. J. Bayarri, J. O. Berger, A. P. Dawid, D. Heckerman, A. F. M. Smith, and M. West, Oxford: Oxford University Press. [4]

Gramacy, R., and Polson, N. (2011), "Particle Learning of Gaussian Process Models for Sequential Design and Optimization," Journal of Computational and Graphical Statistics, 20, 102-118. [4]

Huang, D., Allen, T., Notz, W., and Zeng, N. (2006), "Global Optimization of Stochastic Black-Box Systems via Sequential Kriging Meta-Models," Journal of Global Optimization, 34, 441-466. [2,3,4,5,8]

Humphrey, D., and Wilson, J. (2000), "A Revised Simplex Search Procedure for Stochastic Simulation Response Surface Optimization," INFORMS Journal on Computing, 12, 272-283. [8]

Jones, D., Schonlau, M., and Welch, W. (1998), "Efficient Global Optimization of Expensive Black-Box Functions," Journal of Global Optimization, 13, 455-492. [2,4]

Kennedy, M., and O'Hagan, A. (2000), "Predicting the Output From a Complex Computer Code When Fast Approximations are Available," Biometrika, 87, 1-13. [3]

Mockus, J. (1988), Bayesian Approach to Global Optimization, Amsterdam: Kluwer. [7]

Qian, P., and Wu, C. (2008), "Bayesian Hierarchical Modeling for Integrating Low-Accuracy and High-Accuracy Experiments," Technometrics, 50, 192-204. [3]

Rasmussen, C., and Williams, C. (2006), Gaussian Processes for Machine Learning, Cambridge, MA: The MIT Press. [3]

Roustant, O., Ginsbourger, D., and Deville, Y. (2011), DiceKriging: Kriging Methods for Computer Experiments, R Package Version 1.3.2, Vienna: R Foundation for Statistical Computing. [9,11]

Schonlau, M., Welch, W., and Jones, D. (1998), "Global Versus Local Search in Constrained Optimization of Computer Models," Lecture Notes-Monograph Series, 34, 11-25. [5]

\title{
Comment: Properties and Practicalities of the Expected Quantile Improvement
}

\author{
Alexander I. J. FoRRESTER \\ Faculty of Engineering \\ and the Environment \\ University of Southampton \\ Southampton SO17 1BJ, UK \\ (Alexander.Forrester@soton.ac.uk)
}

The expected quantile improvement (EQI) of Picheny et al. (2013) is an extension of the expected improvement (EI) sequential sampling criterion for selecting experiments that can be performed at varying levels of accuracy (yielding varying degrees of noise). It is an elegant formulation that reduces to the original EI in the absence of noise. This comment will first examine the properties of the EQI, following on from the discussion in Picheny et al. The effect of each control parameter, which essentially determines to what extent the EQI departs from the EI, is briefly investigated using a one-dimensional test function. Following this I will consider the EQI in the context of solving practical engineering problems; looking at the assumptions made about the convergence properties of simulators, which are key to the success of the method, and at implementa- tion issues, including the efficient application of computational resources.

\section{PROPERTIES OF THE EQI}

The EQI depends on the choice of quantile $\beta$, the estimate of the future noise $\tau_{n+1}^{2}$, the estimates of the kriging hyperparameters, and the past noise $\tau_{1, \ldots, n}^{2}$. The past noise is not necessarily

C 2013 American Statistical Association and the American Society for Quality TECHNOMETRICS, FEBRUARY 2013, VOL. 55, NO. 1 DOI: $10.1080 / 00401706.2012 .733322$ 